\title{
An Overview of Spiritual Health in Cancer Patients
}

\author{
Fatemeh Samiee $\operatorname{Rad}^{1}$, Mehri Kalhor ${ }^{2, *}$
}

\begin{abstract}
${ }^{1}$ Associate Professor of Pathology Department, Metabolic Diseases Research Center, Qazvin University of Medical Sciences. Qazvin, Iran

${ }^{2} \mathrm{PhD}$ Student of Reproductive Health of Tarbiat Modares University, Kosar Hospital, Qazvin University of Medical Sciences. Qazvin, Iran

* Corresponding author: Mehri Kalhor, PhD Student of Reproductive Health of Tarbiat Modares University, Kosar Hospital, Qazvin University of Medical Sciences. Qazvin, Iran. Email: mehrikalhor@modares.ac.ir
\end{abstract}

Received: 29 Jul 2017

Accepted: 30 Oct 2018

Abstract
Introduction: Spirituality is one of the important dimensions of human. Spiritual crises can have an adverse
effect on physical and mental health. Diseases cause spiritual distress in patients. This is important in patients
with chronic illness, such as cancer. The purpose of this study is to provide an overview of the spiritual health
of cancer patients.
Methods: This descriptive-prospective study was conducted on 1442 patients with cancer in the pathology
department of Qazvin Hospitals from 2011 to 2015 by census method. The tool used in research was
Ellison's Pallutinin Spiritual Health Questionnaire. Descriptive statistics and Pearson test were used to
determine the correlation between variables $P<0.05$.
Results: The average age patients was $56 \pm 66.74$ (age range 26 to 81 ). 850 ( $58 \%$ ) patients were male. The
results showed that spiritual health level of $21 \%$ of patients were at intermediate level, 55 percent on average,
and 23 percent were at a high level. Religious health scores of the patients were higher than that of health
existence. Between demographic variables and spiritual health ( $\mathrm{P}=0.65$ ) no significant association was not
found. But between the rate of cancer progression and spiritual health, statistically significant correlation was
found (P = 0.04).
Conclusions: The present study shows that most cancer patients have a moderate spiritual level. Also, the
level of religious health was higher than existential health. Therefore, paying attention to spirituality in
patients with chronic illness is important and it is necessary for health providers to be familiar with the
psychic and spiritual factors of patients.
Keywords: Spirituality, Cancer, Mental Health




\section{نماى كلى از سلامت معنوى در بيمار ان سرطانى}

\section{فاطمه سميعىراد '، مهرى كلهر}

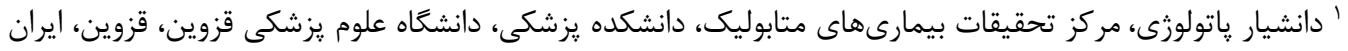

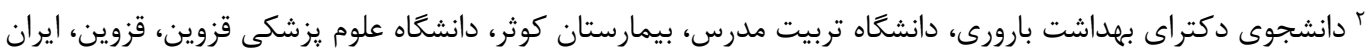

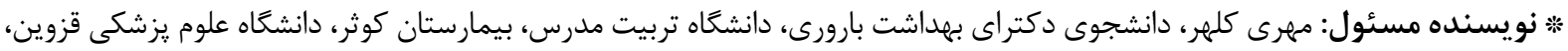
قزوين، ايران. ايميل: mehrikalhor@modares.ac.ir

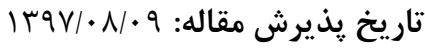

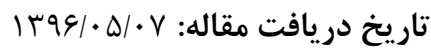

جكيده

مقدمه: معنويت يكى از ابعاد مهمم انسان است. بحر انهاى معنوى مى تواند داراى اثر نامطلوب درسلامت جسمى و روحى باشند. بيمارىها

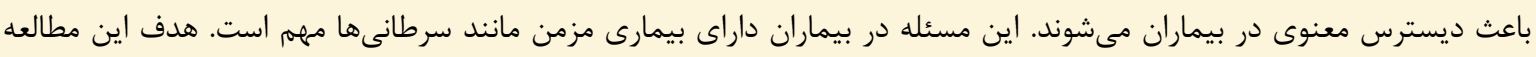

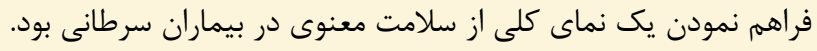

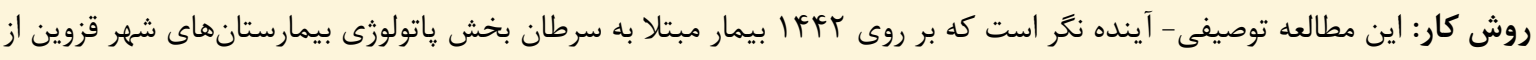

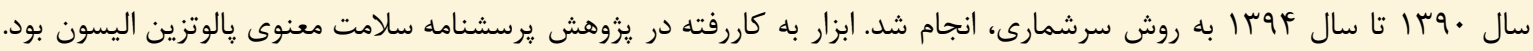

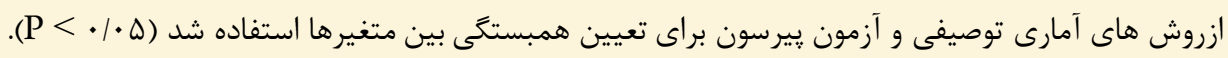

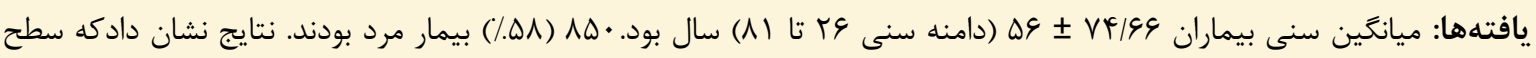

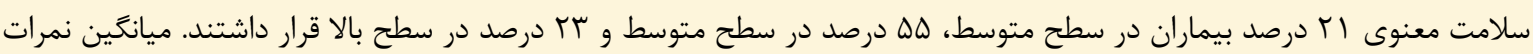

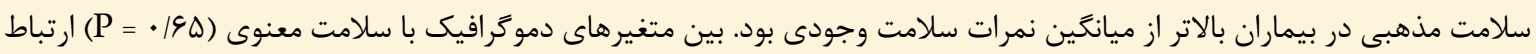

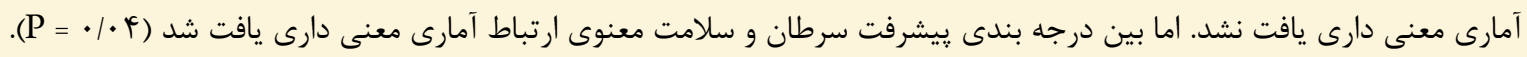

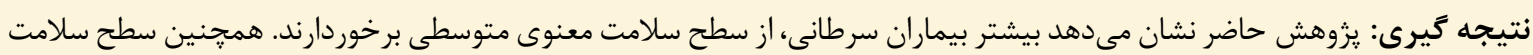

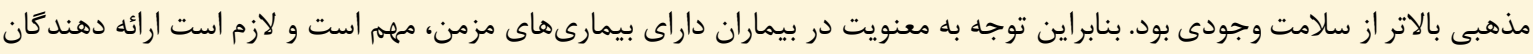
سلامت با عوامل روحى و معنوى بيماران آشنا باشند.

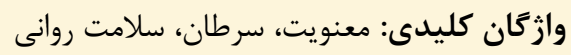

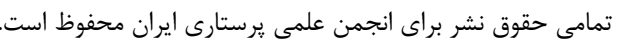

مقدمه

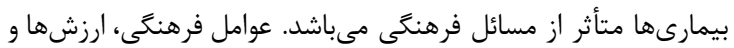

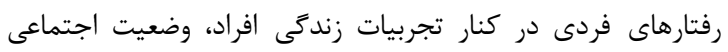

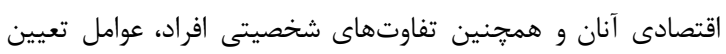

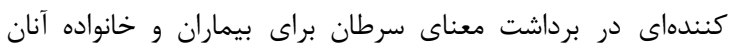

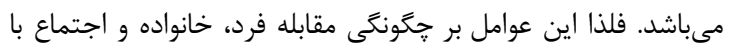

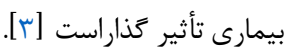

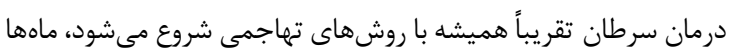

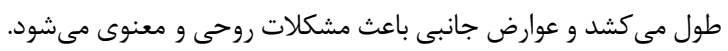

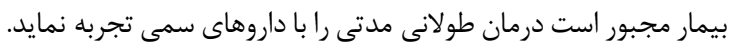

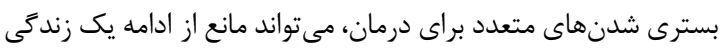

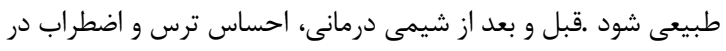

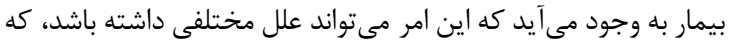

سرطان، يكى از بيمارى هاى شايع مزمن و غيرواكير است. سرطانها علت

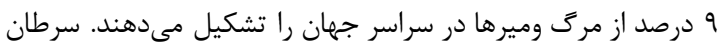

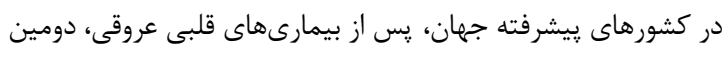

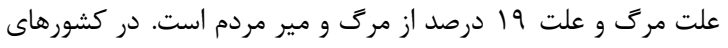

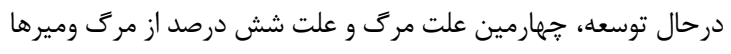

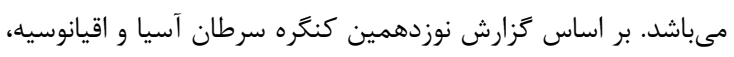

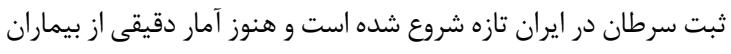

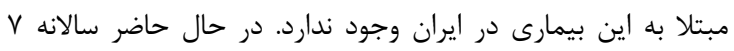

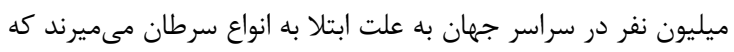

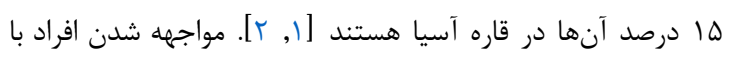

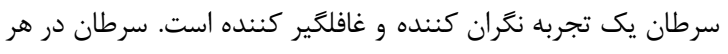

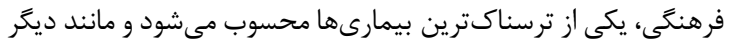


خلق معنا در زندگى و الهام بخش فرد در رويارويى با مشكلات ميشود .

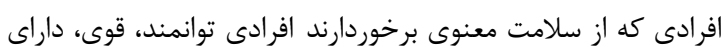

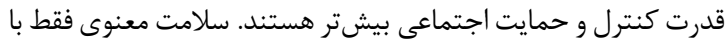

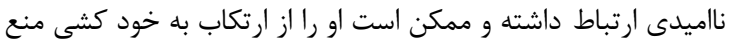

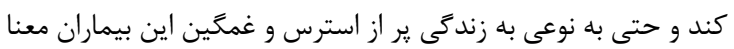

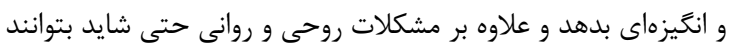

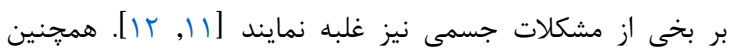

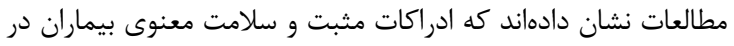

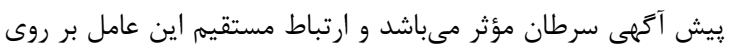

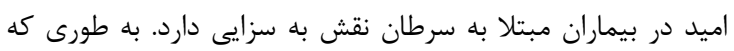

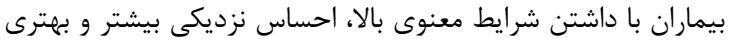

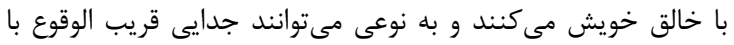

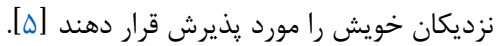

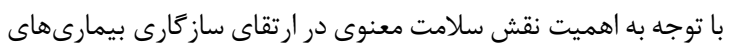

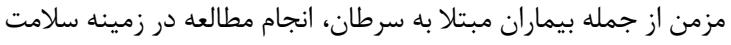

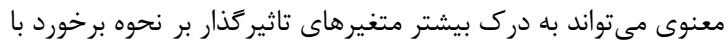

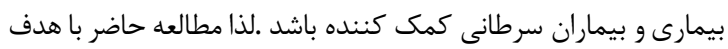

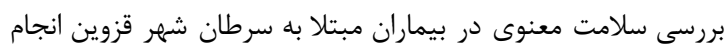

\section{روش كار}

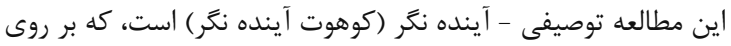

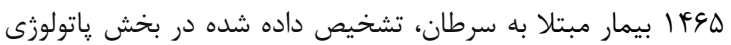

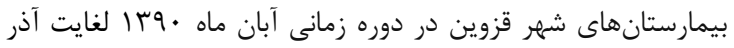

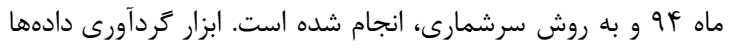

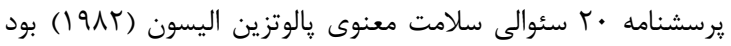

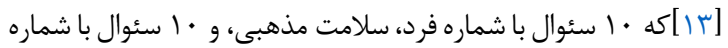

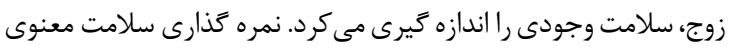

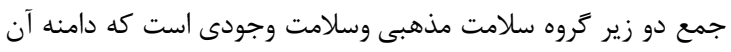

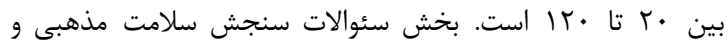

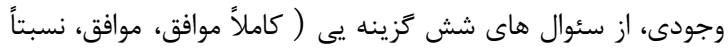

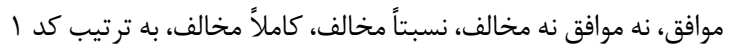

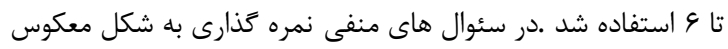

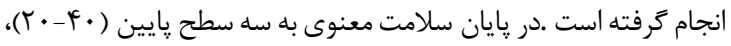

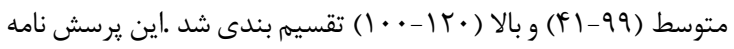

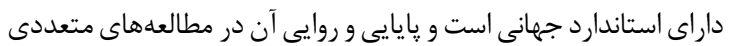

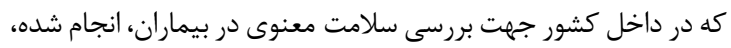

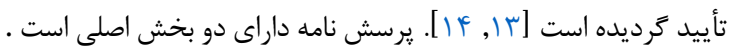

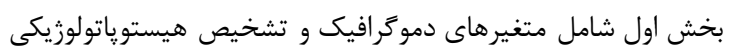

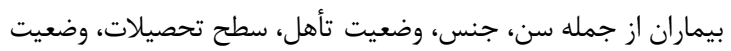

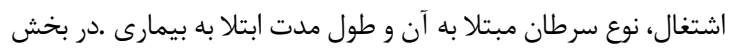

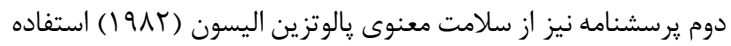

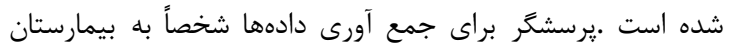

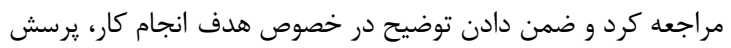

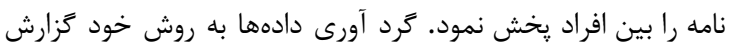

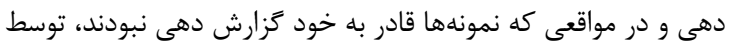

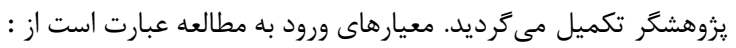

بعضى از آنها شامل :عوارض ناشى از شيمى درمانى، سوراخ كردن وريد

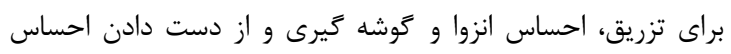

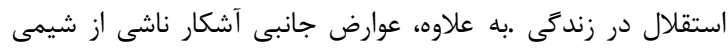

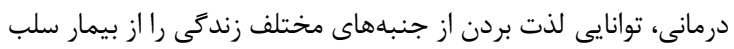

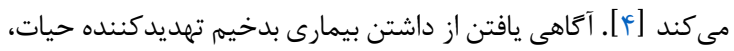

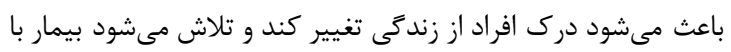

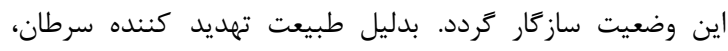

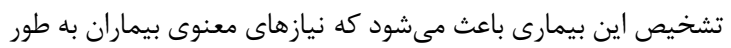

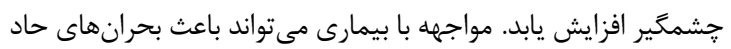

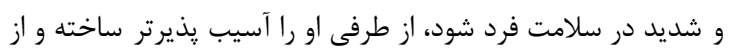

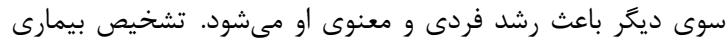

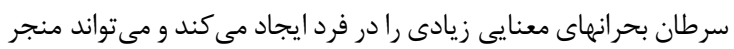

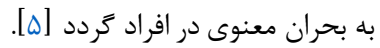

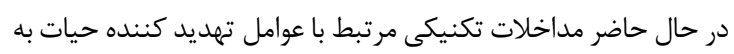

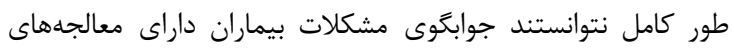

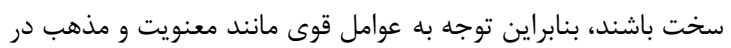

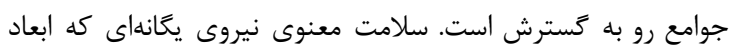

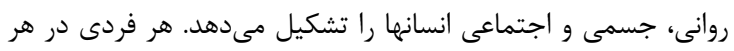

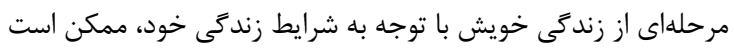

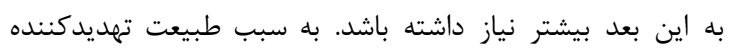

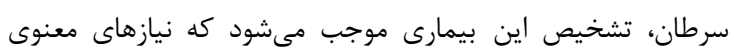

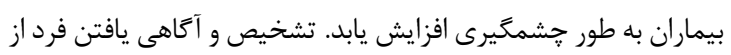

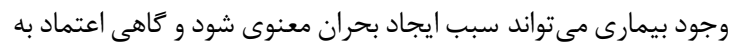

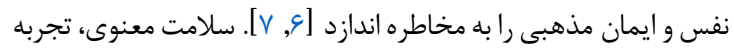

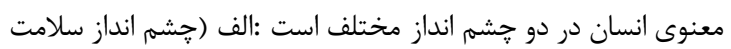

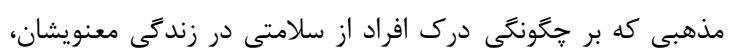

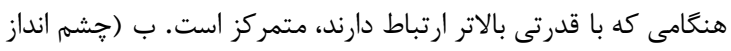

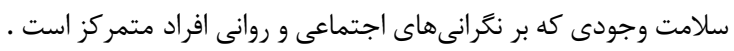

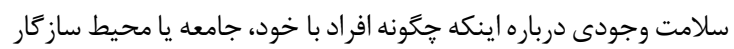

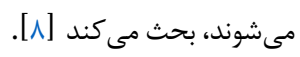

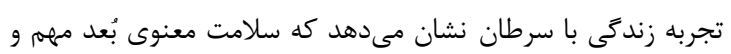

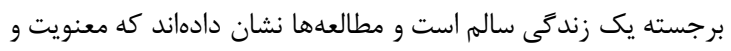

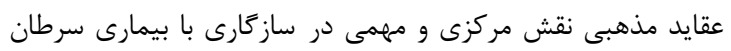

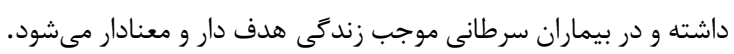

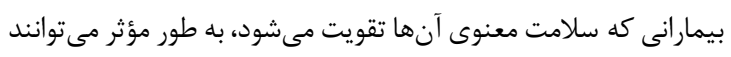

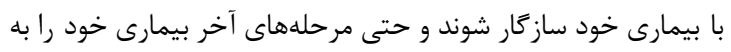

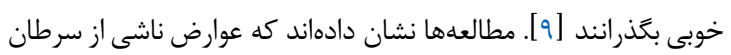

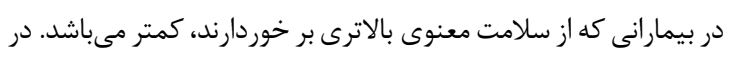

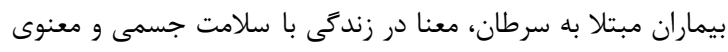

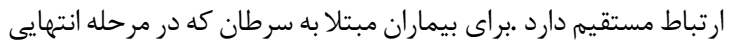

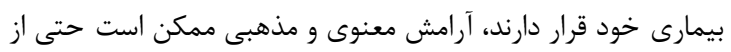

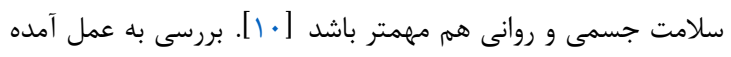

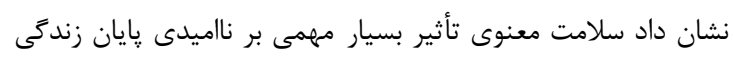

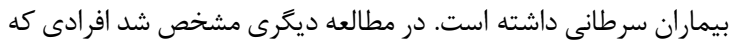

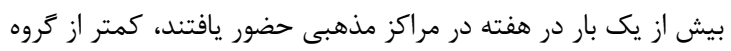

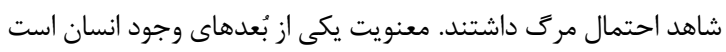

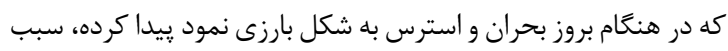


نظر نوع سرطان، VY درصد سرطان دستخاه گوارش، ه/ه درصددستگاه

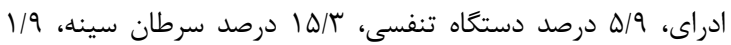

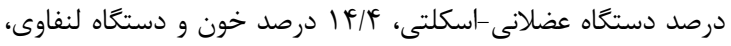

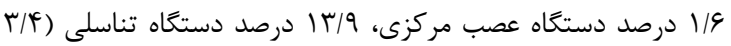

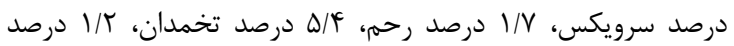

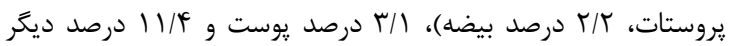

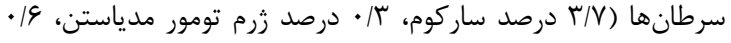

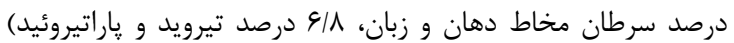

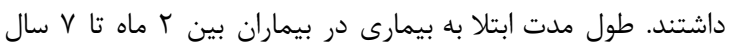

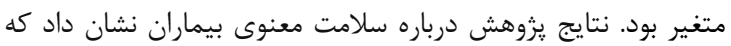

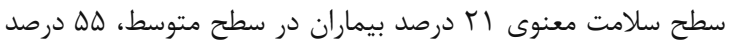

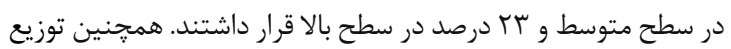
فراوانى و درصد دو بعد سلامت معنوى (مذهبى و وجودى) نشان داده

شد. (جدول (1).

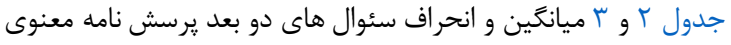

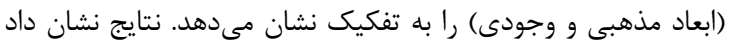

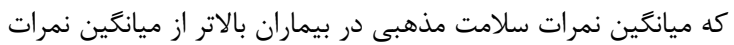

سلامت وجودى بود.
داشتن حداقل \\ سال سن، تمايل به همكارى آكاهى از نوع بيمارى

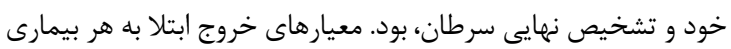

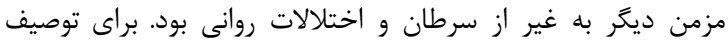

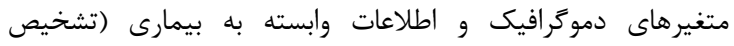

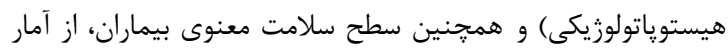

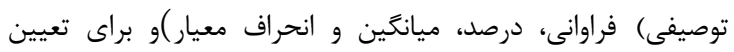

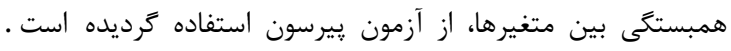

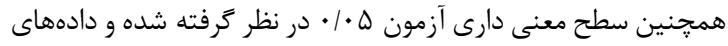

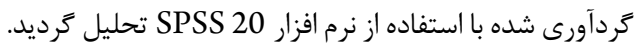

\section{كافتهها}

نتايج مطالعه نشان داد كه ميانگين سنى

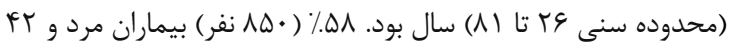

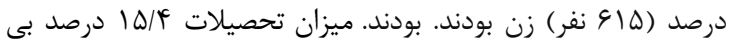

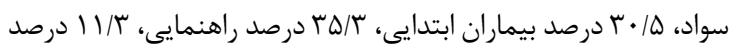

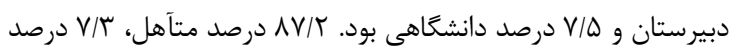

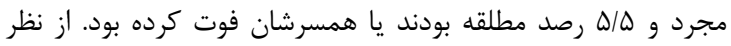

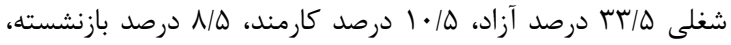

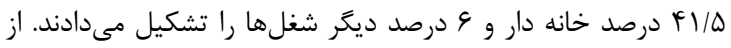

\begin{tabular}{|c|c|c|c|}
\hline & \multicolumn{3}{|c|}{ حدول ا: نمرههاى سلامت معنوى در بيماران مبتلا به سرطان } \\
\hline نمره كل (سلامت معنوى) & سلامت وجودى & سلامت مذهبى & سطح سلامت \\
\hline فراوانى (درصد) & فراوانى (درصد) & فراوانى (درصد) & \\
\hline$(/ / r 1) r \cdot V$ & $(/ . \Lambda) \mid r \cdot$ & $(/ .1 \Gamma) \backslash \wedge \vee$ & ضعيف (•r-F) \\
\hline$(/ . \Delta \Delta) \Lambda \cdot \Delta$ & (\%THE & $(/ . r T) \& V$. & متوسط (q)-99) \\
\hline$(/$ TYATAL & $(/ 1 \cdot) \mid \Delta r$ & $(/ .14) \Gamma \cdot \cdot$ & 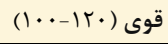 \\
\hline
\end{tabular}

جدول r: شاخصهاى توصيفى (ميانكين و انحراف معيار) براى نمرات سلامت مذهبى

انحراف معيار \pm ميانگين

$\Delta / r \pm 1 / 1$

$\Delta / V \pm \cdot / \Lambda$

$\Delta / r \pm 1 / 4$

$\Delta / \Lambda \pm \cdot / 9$

$\varphi / V \pm 1 / r$

$\Delta / 9 \pm \cdot / 9$

$r / \Lambda \pm 1 / 9$

$r / 9 \pm 1 / 1$

$\Delta / r \pm 1 / \cdot r$

$\Delta / \Delta \pm 1 / \cdot 1$
سؤال هاى مذهبى

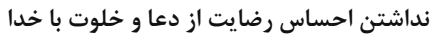

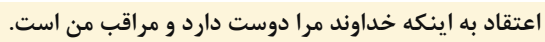

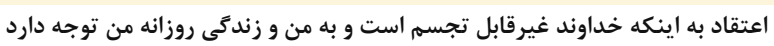
برقرارى ارتباط معنادار با خدا

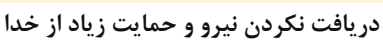
باور به اينكه خداوند به فكر مشكل دهاى من است

نداشتن ارتباط فردى رضايت بخش با خداوند.

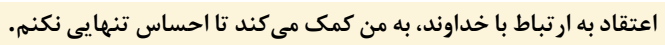
احساس كمال هنغًام رابطه صميمى و نزديك با خداوند.

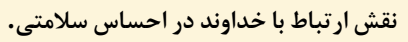

جدول با: شاخصهاى توصيفى (ميانَين و انحراف معيار) براى نمرات سلامت وجودى

انحراف معيار \pm ميانگين

$F / V \pm I / V$

$F / \Delta \pm 1 / \Delta$

$\varphi / r \pm 1 / 9$

$r / r \pm 1 / \Lambda$

$r / 9 \pm r / \cdot 1$

$F / T \pm 1 / 9$

$\varphi / l \pm 1 / V$

$F / \Lambda \pm 1 / \cdot 1$

$f / 9 \pm 1 / V$

$r / 9 \pm 1 / 9$
سؤال هاى وجودى آنساي

نمىدانم كه هستم، از كجا آمدهام و به كجا خودى آهم اهم رفت.

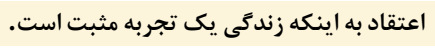
احساس مى كنم آينده نامعلومى دارم. رسيدن به كمال در زندكى و رضايت از زندكى احند.

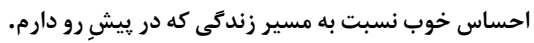
لذت نبردن از زندكى. احساس خوب نسبت به آينده. احساس مى كنم زندكى ير از ناملايمات و ناخوشى ها است بـ آنده. بعى معنايى زندكى. اعتقاد به وجود هدفى خاص براي زنى زنده بودن. 
و آنان اعتقاد داشتند كه براى احساس راحتى بيشتر و كسب توان دوباره

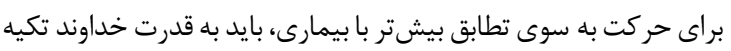

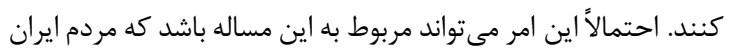

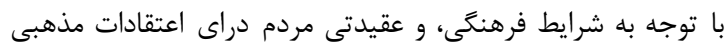

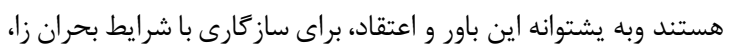

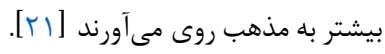

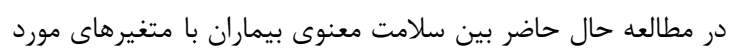

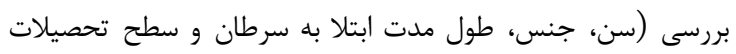

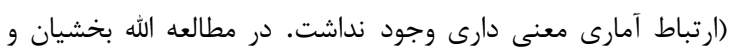

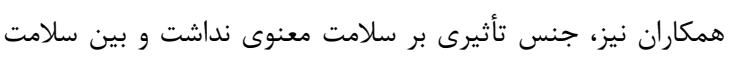

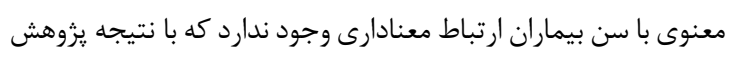

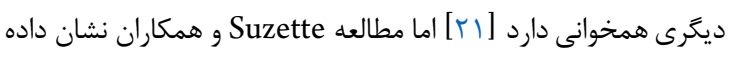

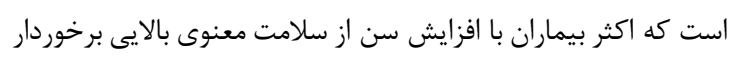

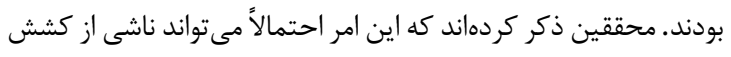

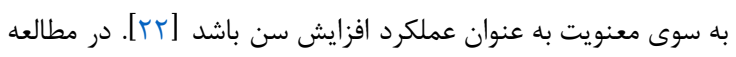

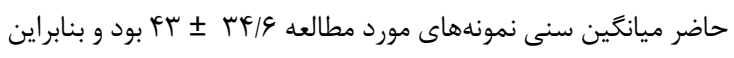

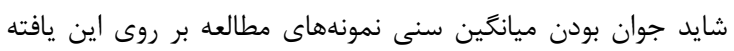

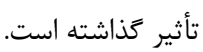

نتيجههاى يافتههاى Hatamipour نيز هيج ارتباطى را بين سين سطح

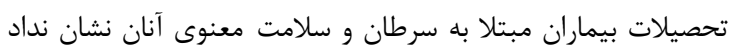

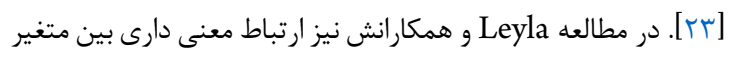

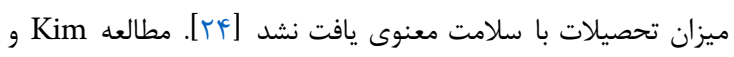

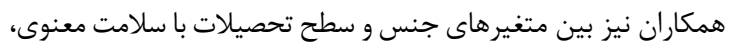

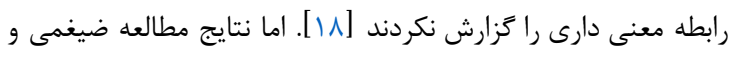

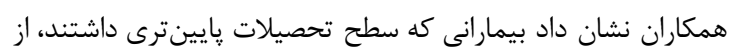

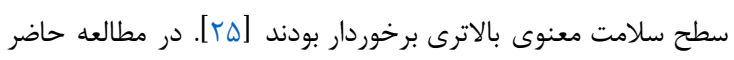

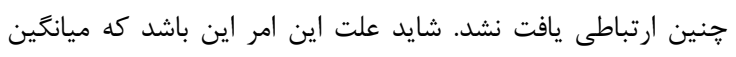

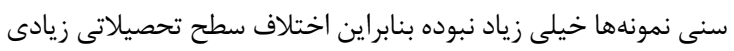

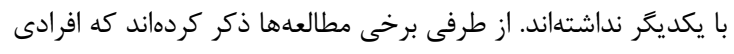

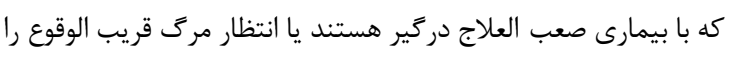

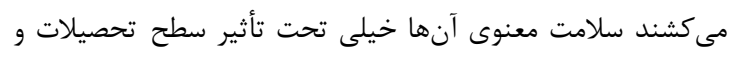
وضعيت اقتصادى قرار نمى كيرد.

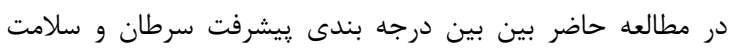

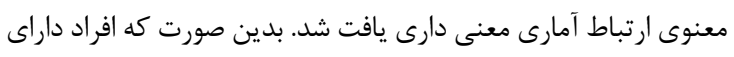

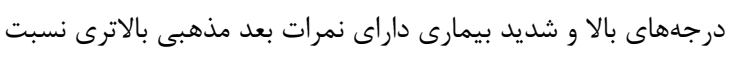

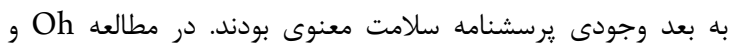

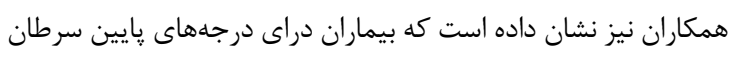

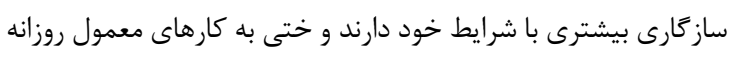

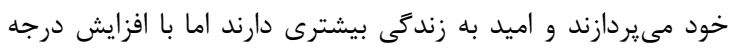

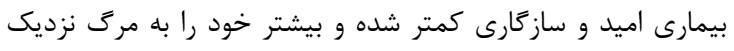

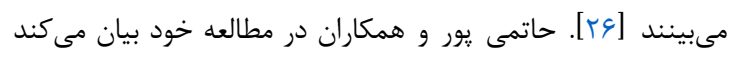

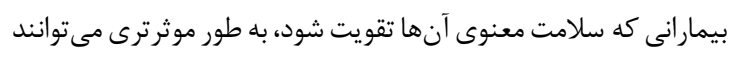

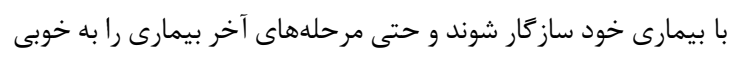

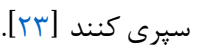

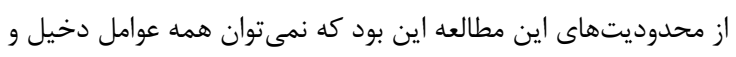

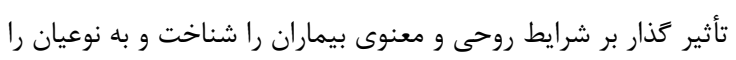

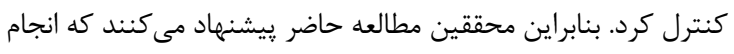

همجنين آزمونهاى آمارى براى بررسى ارتباط بين متغيرهاى

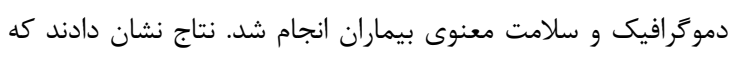

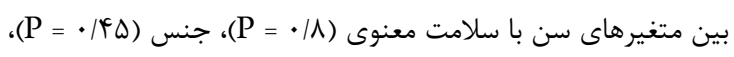

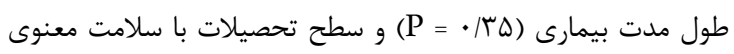

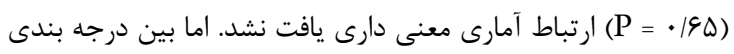

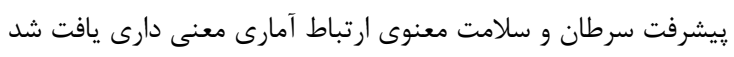
. $(\mathrm{P}=\cdot / \cdot \varphi)$

نتايج يُوهش حاضر نشان داد كه سطح سلامت معنوى اكثر واحدهاى

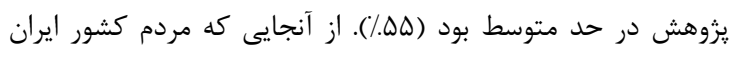

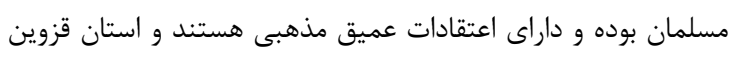

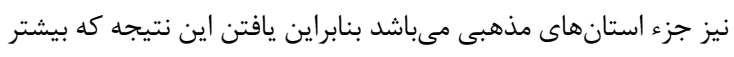

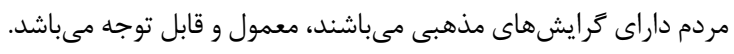

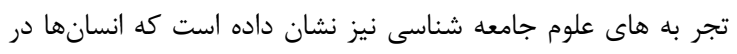

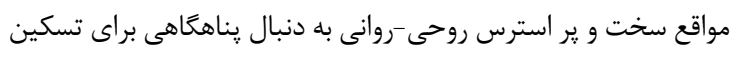

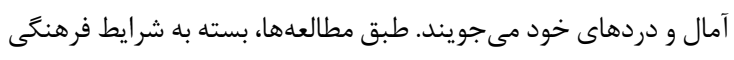

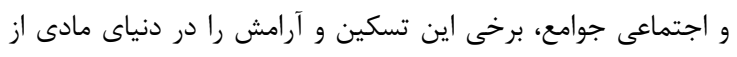

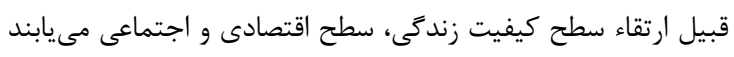

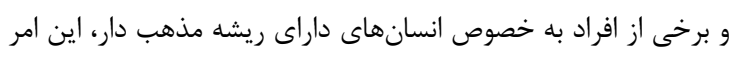

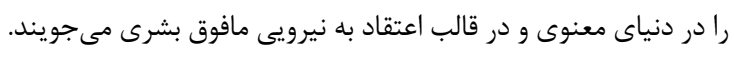

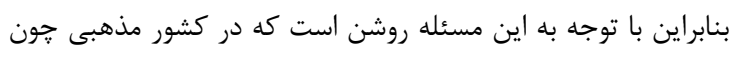

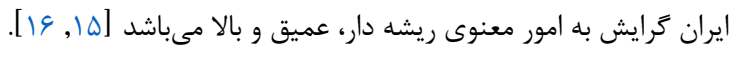

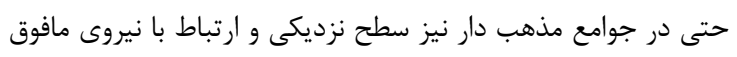

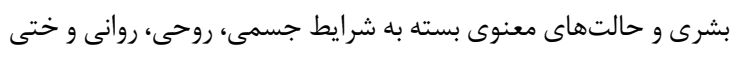

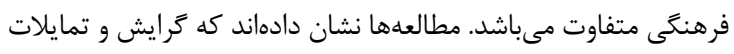

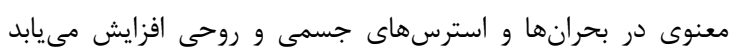

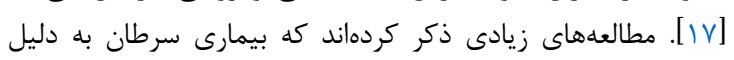

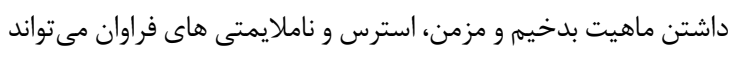

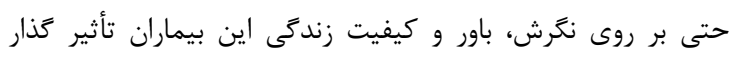

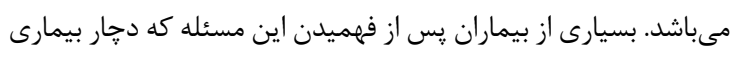

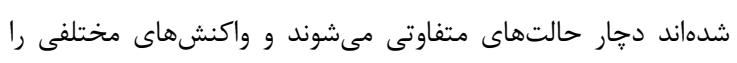

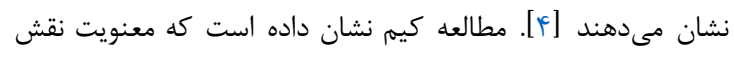

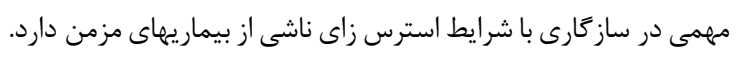

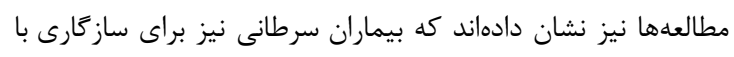

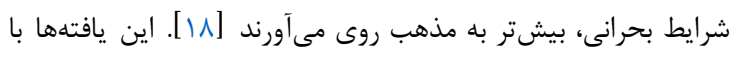

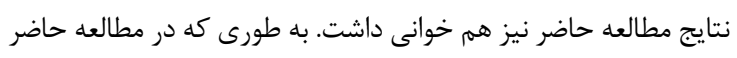

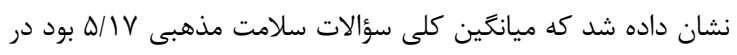

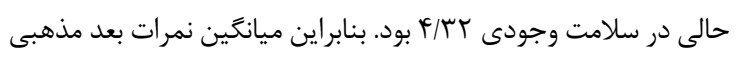

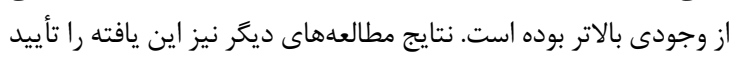

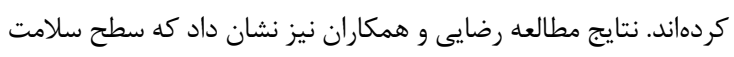

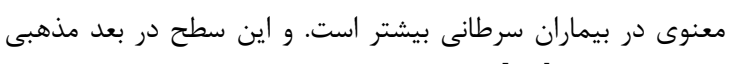

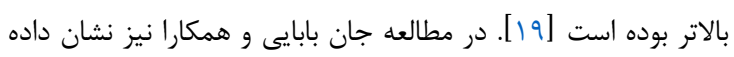

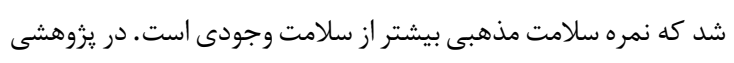

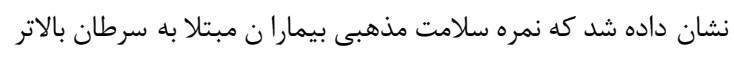

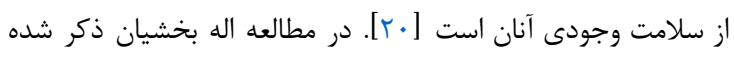

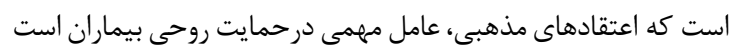




\section{نتيجه تيرى}

يزوهش حاضر نشان مى دهد بيشتر بيماران سرطانى، از سطح سلامت

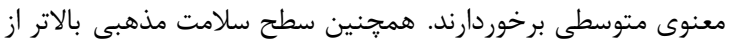

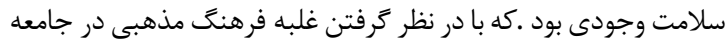

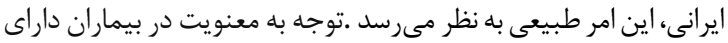

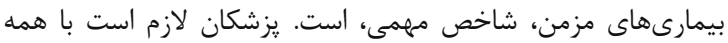

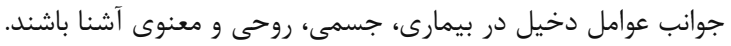

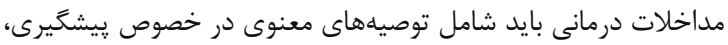

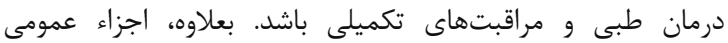
توصيههاى معنوى بايد در غربالكرىهاى روتين يزشكى، لحاظ كردد.

بدين وسيله محققين از همكارى صميمانه بيمارانى كه با مطالعه حاضر همكارى داشتند، نهايت قدردانى و سياس راسئه را دارند. تضادمنافع

نويسندًان مقاله اعلام مىدارند كه در نعارش اين مقاله هيج گونه تضاد

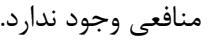

\section{References}

1. Siegel RL, Miller KD, Jemal A. Cancer statistics, 2016. CA Cancer J Clin. 2016;66(1):7-30. doi: 10.3322/caac.21332 pmid: 26742998

2. Dasgupta P, Turrell G, Aitken JF, Baade PD. Partner status and survival after cancer: A competing risks analysis. Cancer Epidemiol. 2016;41:16-23. doi: 10.1016/j.canep.2015.12.009 pmid: 26775082

3. Afrooz R, Rahmani A, Zamanzadeh V, Abdullahzadeh F, Azadi A, Faghany S, et al. The nature of hope among Iranian cancer patients. Asian Pac J Cancer Prev. 2014;15(21):9307-12. pmid: 25422217

4. Abdollahzadeh F, Moradi N, Pakpour V, Rahmani A, Zamanzadeh V, Mohammadpoorasl A, et al. Un-met supportive care needs of Iranian breast cancer patients. Asian Pac J Cancer Prev. 2014;15(9):3933-8. pmid: 24935576

5. Seyedrasooli A, Rahmani A, Howard F, Zamanzadeh V, Mohammadpoorasl A, Aliashrafi R, et al. Iranian cancer patient perceptions of prognosis and the relationship to hope. Asian Pac J Cancer Prev. 2014;15(15):6205-10. pmid: 25124599

6. Harding R, Powell RA, Namisango E, Merriman A, Gikaara N, Ali Z, et al. Palliative care-related self-report problems among cancer patients in East Africa: a twocountry study. Support Care Cancer. 2014;22(12):318592.

7. Ng GC, Mohamed S, Sulaiman AH, Zainal NZ. Anxiety and Depression in Cancer Patients: The Association with Religiosity and Religious Coping. J Relig Health. 2017;56(2):575-90. doi: 10.1007/s10943-016-0267-y pmid: 27287259

8. Rabow MW, Knish SJ. Spiritual well-being among outpatients with cancer receiving concurrent oncologic and palliative care. Support Care Cancer. 2015;23(4):919-23.

$$
\begin{aligned}
& \text { مطالعاتى اين خنين به صورت مصاحبههاى كيفى عميق براى دست } \\
& \text { يابى به شرايط عميقتر شرايط روحى و معنوى بيماران انجام كيرد. }
\end{aligned}
$$

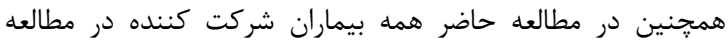

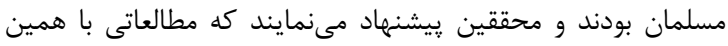

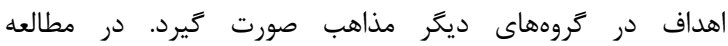

$$
\begin{aligned}
& \text { Seyedrasooli }
\end{aligned}
$$

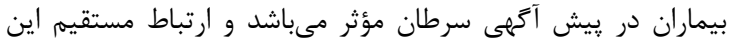

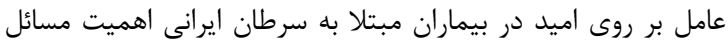

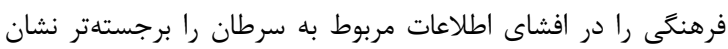

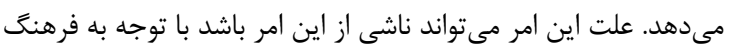

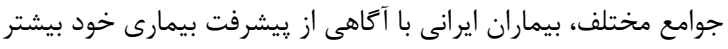

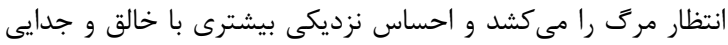

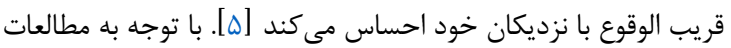

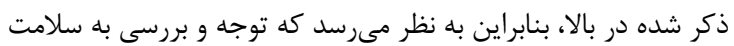

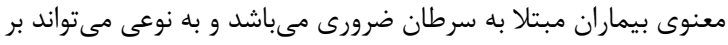

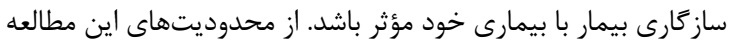

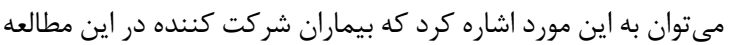

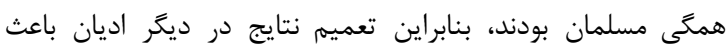

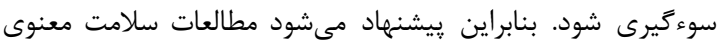

$$
\begin{aligned}
& \text { كيفى و نيزمطالعه در جوامع داراى اديان ديكر نيز انجام شود. }
\end{aligned}
$$

9. Seyedrasooly A, Rahmani A, Zamanzadeh V, Aliashrafi Z, Nikanfar A-R, Jasemi M. Association between perception of prognosis and spiritual well-being among cancer patients. J Caring Sci 2014;3(1):47.

10. Akalin A, Pinar G. Unmet needs of women diagnosed with gynecologic cancer: an overview of literature. Palliat Care Med. 2016;6(2):1-6. doi: 10.4172/2165-7386.1000249

11. Hekmati Pour N, Hojjati H. The relationship between praying and life expectancy in cancerous patients. J Med Life. 2015;8(4):60-4

12. Ebrahimi H, Ashrafi Z, Eslampanah G, Noruzpur F. Relationship between spiritual well-being and quality of life in hemodialysis patients. J Nurs Midwifery Sci 2014;1(3):41-8. doi: 10.18869/acadpub.jnms.1.3.41

13. Aghahoseini S, Rahmani A, Abdollahzadeh F, Kermani I. Spiritual health of cancer patients and its related factors. Qom J Res Med Sci 2009;5(3):7-12.

14. Rodger KB. The Spiritual Well-Being Scale. J Psychol Theol. 1991;19(1):56-70. doi: $10.1177 / 009164719101900106$

15. Sajedi A, Namazi M. [Roundtable weakening and strengthening of cultural self and non-self causes of youth culture]. Marifat Monthly. 2000;45(13).

16. Rahimi $M$. The relationship between spiritual intelligence of students and their tendency to groups of Stupor and Satanism. Discipl Knowledge Q Chaharmahal Bakhtiari. 2012;1(2).

17. Shankar S. Spiritual intelligence: An overview. Int J Multidiscipl Res Dev. 2016;3(3):224-7.

18. Kim JH, McMahon BT, Hawley C, Brickham D, Gonzalez $\mathrm{R}$, Lee DH. Psychosocial Adaptation to Chronic Illness and Disability: A Virtue Based Model. J Occup Rehabil. 2016;26(1):45-55. doi: 10.1007/s10926-015-9622-1 pmid: 26781509 
19. Rezaie A, Momeni Ghaleghasemi T, Ebrahimi A. The Relationship between Spiritual Wellbeing with Stress, Anxiety, and Some Demographic Variables in Women with Breast Cancer Referring to the Specialized Cancer Treatment Center in Isfahan, Iran. J Health Syst Res. 2012;8(1):104-13.

20. G J, Esmaeili Ravanbakhsh B, N M-S. A survey of the role of spiritual health and its related factors in the patient with metastatic digestive cancer. Religion Health. 2014;2(1):914.

21. Allahbakhshian M, Jaffarpour M, Parvizy S, Haghani H. [A Survey on relationship between spiritual wellbeing and quality of life in multiple sclerosis patients]. Zahedan J Med Sci Res. 2011;12(3):29-33.

22. Brémault-Phillips S, Olson J, Brett-MacLean P, Oneschuk $\mathrm{D}$, Sinclair S, Magnus R, et al. Integrating spirituality as a key component of patient care. Religions. 2015;6(2):47698.

23. Hatamipour K, Rassouli M, Yaghmaie F, Zendedel K, Majd HA. Spiritual needs of cancer patients: a qualitative study. Indian J Palliat Care. 2015;21(1):61-7. doi: 10.4103/0973-1075.150190 pmid: 25709188

24. Leyla F, Fatemeh A. Understanding the Role of Spirituality and Faith in Relation to Life Expectancy and End of Life Experience in Terminally-ILL Cancer Patients. Gerontol Geriatric Stud. 2017;1(4):1-10.

25. Zeighamy H, Sadeghi N. Spiritual/religious needs of adolescents with cancer. Religions. 2016;7(7):91. doi: 10.3390/rel7070091

26. Oh PJ, Kim SH. The effects of spiritual interventions in patients with cancer: a meta-analysis. Oncol Nurs Forum. 2014;41(5):E290-301. doi: 10.1188/14.ONF.E290E301 pmid: 25158666 